Supplements to

\title{
Optimal Sales Schemes against Interdependent Buyers
}

\author{
Masaki Aoyagi \\ ISER, Osaka University
}

\section{Property of the Optimal Sequential Scheme}

Under the optimal scheme identified in Theorem 2, the response by each buyer moves in the direction of the response dominant among their predecessors. In other words, a buyer accepts with a higher probability than his predecessor when most buyers before him have chosen to accept, and rejects with a higher probability when most buyers before him have chosen to reject. Formally, the following proposition states that when the state $\alpha_{t-1}>0$ as a result of many buyers having accepted, the expected probability that the next buyer accepts is higher than the probability that the current buyer accepts. Conversely, when the state $\alpha_{t-1}<0$ as a result of many buyers having rejected, the expected probability that the next buyer rejects is higher than the probability that the current buyer rejects.

Proposition S.1. Suppose that every $s_{i}$ has the uniform distribution, that (5) and (6) hold, and that $\underline{s}+c_{0}=0$. Then the following hold under the optimal sequential scheme $\sigma$. For any $\delta>0$, there exists $\bar{\varepsilon}>0$ such that if $\varepsilon<\bar{\varepsilon}$, then $\alpha_{t-1}>\delta$ implies $E^{\sigma}\left[z_{t+1}\left(\tilde{\alpha}_{t}\right) \mid \alpha_{t-1}\right]>z_{t}\left(\alpha_{t-1}\right)$, and $\alpha_{t-1}<-\delta$ implies $E^{\sigma}\left[z_{t+1}\left(\tilde{\alpha}_{t}\right) \mid \alpha_{t-1}\right]<$ $z_{t}\left(\alpha_{t-1}\right)$.

Proof of Proposition S.1 Since $z_{t}$ is an affine function by Theorem A.4, we have (A.29) for any $\alpha$, and hence the expected value of the probability $z_{t+1}$ of acceptance in period $t+1$ conditional on the state $\alpha_{t-1}$ at the beginning of period $t$ equals

$$
E^{\sigma}\left[z_{t+1}\left(\tilde{\alpha}_{t}\right) \mid \alpha_{t-1}\right]=z_{t+1}\left(\alpha_{t-1}\right) .
$$

It hence follows from $a_{t}=\Delta$ and (A.33) that

$$
\begin{aligned}
E^{\sigma}\left[z_{t+1}\left(\tilde{\alpha}_{t}\right) \mid \alpha_{t-1}\right]-z_{t}\left(\alpha_{t-1}\right) & =\frac{1}{2 \Delta}\left\{a_{t+1}-a_{t}+\left(b_{t+1}-b_{t}\right) \alpha_{t-1}\right\} \\
& =\frac{1}{2 \Delta}\left(c_{t+1}-c_{t}\right) \alpha_{t-1}+o(\varepsilon) .
\end{aligned}
$$

Since $c_{t+1}-c_{t}>0$, we obtain the desired result. 


\begin{tabular}{|c|c|c|c|c|}
\hline \multicolumn{4}{|c|}{ Buyer order } & Revenue \\
\hline 1 & 2 & 3 & 4 & 1.0168 \\
\hline 2 & 1 & 3 & 4 & 1.0161 \\
\hline 3 & 1 & 2 & 4 & 1.0124 \\
\hline 4 & 1 & 2 & 3 & 1.0082 \\
\hline
\end{tabular}

Table 1: Uniform Distribution

\section{Optimal Sequential Schemes in Other Environments: Numerical Examples}

In this section, we report results of numerical computation which find that the conclusion of Theorem 2 on the optimal ordering of buyers extends to more general environments. Set the number of buyers $I=4$ and their dependence weights $c_{i}=i / 7$ for $i=1, \ldots, 4$. Our strategy is to use Lemma A.2 to compare the revenue associated with every contingent scheme. That is, we begin with the sequential pricing problems for every pair of buyers and every initial state $\alpha_{0} \in C_{I-2}=C_{2}$, then proceed to the problems with three buyers, and so on. ${ }^{25}$

In the first example, the private signal $s_{i}$ has the uniform distribution over the unit interval, but $c_{i}$ 's do not satisfy (4). We find that the optimal sequential scheme (among all schemes and not just the one with fixed orders) trades with buyers $1, \ldots, 4$ in this order. Table 1 lists the seller's revenue from the four alternative buyer sequences in (A.18).

In the second example, we assume that a buyer's private signal $s_{i}$ has a truncated exponential distribution over $[0, M](M>0)$. Specifically, we suppose that the cumulative distribution $F$ and the associated density $f$ are respectively given by

$$
F\left(s_{i}\right)=\frac{1-e^{-\delta s_{i}}}{1-e^{-\delta M}} \quad \text { and } \quad f\left(s_{i}\right)=\frac{\delta e^{-\delta s_{i}}}{1-e^{-\delta M}} \quad \text { for } s_{i} \in[0, M]
$$

For $M=2$ and $\delta=0.5$, we again find that the optimal sequential scheme trades in the increasing order of the dependence weights. Table 2 lists the seller's revenue from the four buyer sequences in (A.18).

Listed below is the matlab program to compute the seller's revenue along the four alternative sequences in (A.18) for the truncated exponential distribution reported

\footnotetext{
${ }^{25}$ Revenue is computed for each point on the grid of the size $1 / 200 \times 1 / 200$ over the $\left(\alpha_{t-1}, z_{t}\right)$ space for each $t=1, \ldots, 4$. See below for a program source in Matlab for the representative cases.
} 


\begin{tabular}{|c|c|c|c|}
\hline \multicolumn{3}{|c|}{ Buyer order } & Revenue \\
\hline 1 & 2 & 3 & 1.5892 \\
\hline 2 & 1 & 3 & 1.5873 \\
\hline 3 & 1 & 2 & 1.5799 \\
\hline 4 & 1 & 2 & 1.5633 \\
\hline
\end{tabular}

Table 2: Truncated Exponential Distribution

in Table 2. The subroutine follows the main program.

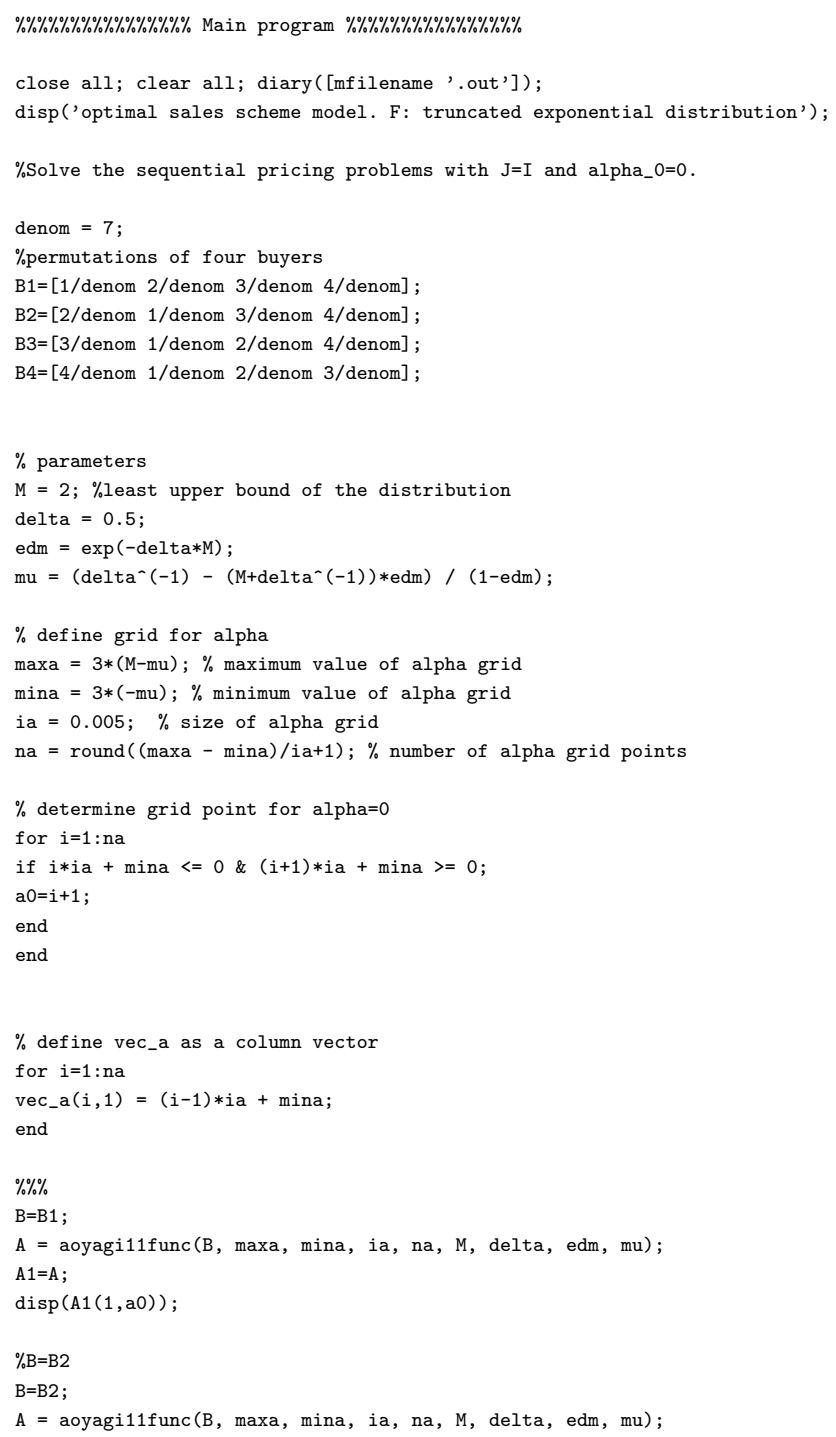


$\mathrm{A} 2=\mathrm{A}$;

$\operatorname{disp}(A 2(1, a 0))$;

$\% \mathrm{~B}=\mathrm{B} 3$

$\mathrm{B}=\mathrm{B} 3$;

$A=\operatorname{aoyagi11func}(B$, maxa, mina, $i a, n a, M$, delta, edm, mu);

$\mathrm{A} 3=\mathrm{A}$;

$\operatorname{disp}(\mathrm{A} 3(1, \mathrm{a} 0))$;

$\% \mathrm{~B}=\mathrm{B} 4$

$\mathrm{B}=\mathrm{B} 4$;

$A=\operatorname{aoyagi11func}(B$, maxa, mina, ia, na, $M$, delta, edm, mu);

$\mathrm{A} 4=\mathrm{A}$;

$\operatorname{disp}(A 4(1, a 0))$;

$\%$ evaluate at the initial state $a=0$

$\operatorname{disp}(' A 1-A 2 ')$;

$\operatorname{disp}(A 1(1, a 0)-A 2(1, a 0))$;

$\operatorname{disp}\left(' A 1-A 3^{\prime}\right)$;

$\operatorname{disp}(A 1(1, a 0)-A 3(1, a 0))$.

$\operatorname{disp}(' A 1-A 4$ ') ;

$\operatorname{disp}(A 1(1, a 0)-A 4(1, a 0))$;

diary off

$\% \% \% \% \% \% \% \% \% \% \% \% \% \% \%$ subroutine $\% \% \% \% \% \% \% \% \% \% \% \% \% \% \% \%$

function $A=\operatorname{aoyagi11func}(B, \operatorname{maxa}$, mina, ia, na, $M$, delta, edm, mu)

$\mathrm{nb}=4 ; \%$ number of buyers

$\%$ define grid for $z$

$\operatorname{maxz}=1 ; \%$ maximum value of $z$ grid

$\operatorname{minz}=0 ; \%$ minimum value of $z$ grid

$i z=0.005 ; \%$ size of $z$ grid

$\mathrm{nz}=\operatorname{round}((\max z-\operatorname{minz}) / i z+1) ; \%$ number of $z$ grid points

$\%$ define $z$ as a column vector

for $i=1: n z$

$z(i, 1)=(i-1) * i z+\min z$

end

$\%$ set parameter values

$\mathrm{cO}=0$;

$\mathrm{ci}=$ ones $(\mathrm{nb}, 1)$;

for $i=1: \mathrm{nb}$;

$c i(i, 1)=B(1, i)$;

end;

$\%$ initialize payoff vectors

$\mathrm{pi}=\operatorname{ones}(\mathrm{nz}, \mathrm{na}) ; \%$ values when $(\mathrm{z}, \mathrm{a})$

pistar $=$ ones(nb,na); $\%$ optimal value when state is a

pistara $=\operatorname{ones}(n z, 1) ; \%$ expected optimal value when accepted

pistarr $=\operatorname{ones}(n z, 1) ; \%$ expected optimal value when rejected

$\% t=4$ start from the final period

$\mathrm{t}=4$

maxat $=(t-1) *(M-m u) ; \%$ maximum of alpha in $t$

minat $=(t-1) *(-\mathrm{mu}) ; \%$ minimum of alpha in $t$

for $i=1$ :na

$a=\operatorname{mina}+(i-1) * i a ; \%$ compute alpha for the $i$ th grid point

for $j=1: n z$; 
theta $=-1 /$ delta $* \log (1-(1-\operatorname{edm}) *(1-\mathrm{z}(j, 1))) ; \%$ theta for each $\mathrm{z}$ edth $=\exp (-$ delta $*$ theta $)$

if a > maxat $\mid$ a $<$ minat $\%$ if the ith grid point exceeds the bounds pi $(:, i)=-100 * n b ; \%$ substitute a small value

else

$p i(j, i)=z(j, 1) *$ theta $+c i(t) * a * z(j, 1)+c 0 * z(j, 1)$

end

end

end

$\operatorname{pistar}(t,:)=\max (p i)$

$\% t=1,2,3$

$t=3$;

while $t>0$

maxat $=(\mathrm{t}-1) *(\mathrm{M}-\mathrm{mu})$

minat $=(\mathrm{t}-1) *(-\mathrm{mu})$;

for $i=1$ : na

$a=\operatorname{mina}+(i-1) * i a$

$\mathrm{k}=\operatorname{ones}(\mathrm{nz}, 1) ; \% \operatorname{kappa}(\mathrm{z})$ as a $\mathrm{nz}$ vector

$1=\operatorname{ones}(n z, 1) ; \% \operatorname{lambda}(z)$ as a $n z$ vector

for $j=1: n z$

theta $=-1 /$ delta $* \log (1-(1-$ edm $) *(1-z(j, 1))) ; \%$ theta for each $z$

edth $=\exp (-$ delta $*$ theta $)$;

if $z(j, 1)==0$

$k(j, 1)=M-m u$

else

$\mathrm{k}(\mathrm{j}, 1)=\left(\left(\right.\right.$ theta $\left.\left.+\operatorname{delta}{ }^{\wedge}(-1)\right) * \operatorname{edth}-\left(M+\operatorname{delta}^{\wedge}(-1)\right) * \operatorname{edm}\right) /($ edth-edm $) \ldots$

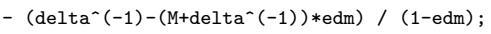

end;

if $z(j, 1)==1$

$l(j, 1)=-m u$;

else

$l(j, 1)=\left(\operatorname{delta}^{\wedge}(-1)-\left(\operatorname{theta}+\operatorname{delta}^{\wedge}(-1)\right) *\right.$ edth $) /(1-$ edth $) \ldots$

- $\left(\operatorname{delta}{ }^{\wedge}(-1)-\left(M+\operatorname{delta}^{\wedge}(-1)\right) *\right.$ edm $) /(1-$ edm $)$;

end;

$a a(j)=a+k(j, 1) ; \%$ future state for each $z$ when accepted

$\operatorname{ar}(j)=a+1(j, 1) ; \%$ future stete for each $z$ when refected

if $\mathrm{aa}(j)>\mathrm{t} *(\mathrm{M}-\mathrm{mu})$;

$\mathrm{aa}(j)=t *(M-m u) ;$

end

if $\operatorname{ar}(j)<t *(-\mathrm{mu})$

$\operatorname{ar}(j)=t *(-m u)$;

end;

gaa $=f i x((a a(j)-$ mina $) / i a)+1 ; \%$ grid point for aa $(j)$

$\operatorname{gar}=f i x((\operatorname{ar}(j)-\operatorname{mina}) / i a)+1 ; \% \operatorname{grid}$ point $f$ or $\operatorname{ar}(j)$

pistara $(j, 1)=$ pistar $(t+1$, gaa $) ; \%$ future payoff pistar_a when accepted $\operatorname{pistarr}(j, 1)=\operatorname{pistar}(t+1, g a r) ; \%$ future payoff pistar_r when rejected

if a $>$ (maxat+ia) $\mid$ a $<$ (minat-ia)

$\mathrm{pi}(j, \mathrm{i})=-100 * \mathrm{nb}$;

else

$p i(j, i)=z(j, 1) *$ theta $+(c i(t) * a+c 0) * z(j, 1)+\ldots$

$z(j, 1) * \operatorname{pistara}(j, 1)+(1-z(j, 1)) * \operatorname{pistarr}(j, 1) ;$

end 
$\operatorname{pistar}(t,:)=\max (p i) ; \%$ choose the maximizing $z$ for each $a$ in period $t$ $\mathrm{t}=\mathrm{t}-1$;

end

$A(1,:)=\operatorname{pistar}(1,:)$; 